(W)

Check for

Cite as

Nano-Micro Lett.

(2021) 13:215

Received: 30 August 2021

Accepted: 25 September 2021

Published online: 21 October 2021

(C) The Author(s) 2021

\section{Electrochemical Surface Restructuring of Phosphorus-Doped Carbon@MoP Electrocatalysts for Hydrogen Evolution}

\author{
Huimin Jiang ${ }^{1,3}$, Liting Yan $^{1}{ }^{凶}$, Shuo Zhang ${ }^{3}$, Yanchao Zhao ${ }^{3}$, Xue Yang ${ }^{1}$, \\ Yameng Wang ${ }^{1}$, Jianxing Shen $^{1}$, Xuebo Zhao ${ }^{1,3}$, Lianzhou Wang ${ }^{2} \bowtie$
}

\title{
HIGHLIGHTS
}

- An electrochemical-induced surface restructuring strategy is developed to design phosphorus-doped carbon@MoP electrocatalysts which exhibits excellent activity for the hydrogen evolution reaction (HER) in both acidic and alkaline electrolytes.

- The activation process and the fundamental mechanism of the prominent synergistic interaction between the phosphorus-doped carbon and MoP are elucidated.

ABSTRACT The hydrogen evolution reaction (HER) through electrocatalysis is promising for the production of clean hydrogen fuel. However, designing the structure of catalysts, controlling their electronic properties, and manipulating their catalytic sites are a significant challenge in this field. Here, we propose an electrochemical surface restructuring strategy to design synergistically interactive phosphorus-doped carbon@MoP electrocatalysts for the HER. A simple electrochemical cycling method is developed to tune the thickness of the carbon layers that cover on MoP core, which significantly influences HER performance. Experimental investigations and theoretical calculations indicate that the inactive surface carbon layers can be removed through electrochemical cycling, leading to a close bond between the $\mathrm{MoP}$ and a few layers of coated graphene. The electrons

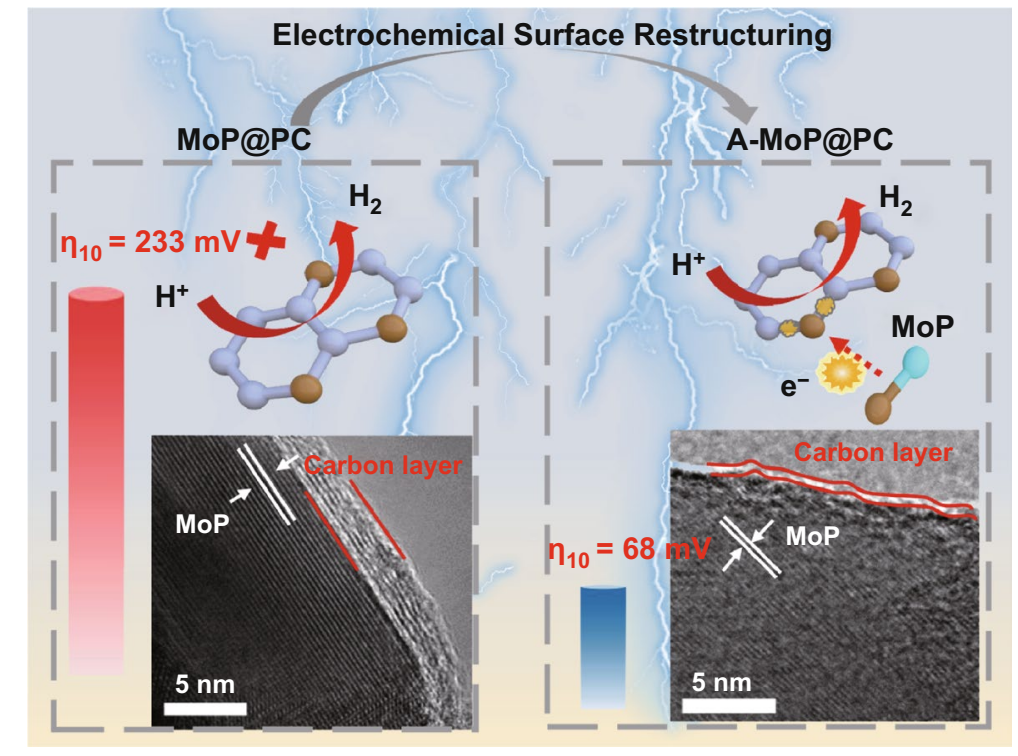
donated by the MoP core enhance the adhesion and electronegativity of the carbon layers; the negatively charged carbon layers act as an active surface. The electrochemically induced optimization of the surface/interface electronic structures in the electrocatalysts significantly promotes the HER. Using this strategy endows the catalyst with excellent activity in terms of the HER in both acidic and alkaline environments (current density of $10 \mathrm{~mA} \mathrm{~cm}^{-2}$ at low overpotentials, of $68 \mathrm{mV}$ in $0.5 \mathrm{M} \mathrm{H}_{2} \mathrm{SO}_{4}$ and $67 \mathrm{mV}$ in $1.0 \mathrm{M} \mathrm{KOH}$ ).

KEYWORDS Electrochemical surface restructuring; Hydrogen evolution; Molybdenum phosphide nanowires; Phosphorus-doped carbon; Synergistic interaction

$\triangle$ Liting Yan, yanlt@qlu.edu.cn; Xuebo Zhao, zhaoxuebo@upc.edu.cn; Lianzhou Wang, l.wang@uq.edu.au

1 School of Materials Science and Engineering, Qilu University of Technology (Shandong Academy of Sciences), No. 3501, Daxue Road, Changqing District, Jinan 250353, People's Republic of China

2 Nanomaterials Centre, School of Chemical Engineering and Australian Institute for Bioengineering and Nanotechnology, The University of Queensland, St Lucia, QLD 4072, Australia

3 College of Chemical Engineering, China University of Petroleum (East China), Huangdao District, No. 66, West Changjiang Road, Qingdao 266580, People's Republic of China 


\section{Introduction}

The development of advanced materials for renewable energy applications has attracted increasing research attention in recent years [1-4]. As an abundant, zero carbon emission source and renewable energy carrier, hydrogen plays an important role in sustainable energy and industrial chemical systems [5-8]. Hydrogen production through electrochemical water-splitting is a type of hydrogen-production technology that is advantageous owing to its high energy-conversion rate and environmentally friendly process [9-13]. Developing high-performance, low-cost electrocatalysts is an ideal method for solving the current energy crisis [14-18].

To date, transition-metal phosphides (TMPs) have already been widely used as active and low-cost hydrogen evolution reaction (HER) electrocatalysts [19-22]. Among the myriad existing TMPs, molybdenum phosphide (MoP) exhibits great potential for application owing to its intrinsic catalytic property [23-26]. However, the performances of TMPs cannot be fully maximized owing to the poor conductivity and the harsh conditions in strongly acidic or alkaline media, along with the fact that the high overpotential in electrocatalysis leads to corrosion, agglomeration, or oxidation. Therefore, maintaining the high stability of TMPs with the simultaneous enhancement of their activity under harsh conditions is a crucial challenge. Recently, encapsulating transition-metal nanoparticles with stable carbon layers has emerged as a novel strategy for designing active and durable catalysts [27, 28]. The ultrathin graphene shell significantly promotes electron transfer from the encapsulated metals to the graphene surface, which optimizes the electronic structure of the graphene surface and triggers the catalytic activity of the inert graphene surface. The synergistic effect of the metal core and carbon layer is strongly related to the thickness of the carbon layer. Density functional theory (DFT) calculations regarding the effect of the thickness of the carbon layer on its performance showed that the carbon shell surface was not likely to be activated if the layer number was greater than three [29]. However, it is difficult to control the thickness of the carbon layers, the unpredictable carbon layers that cover active species are often too thick, which could significantly reduce the catalytic activity.

Recent research on the activity of the HER and oxygen evolution reaction (OER) has suggested that the in situ reconstructed surface of catalysts under electrochemical conditions provides intrinsic active sites for high catalytic activities [30]. For example, Liu et al. discovered that phosphides derived from CoFeO@ black phosphorus (BP) formed during HER catalysis [31]. Driess et al. found that the surface reconstruction led to lattice vacancies and defects due to the loss of $\mathrm{Li}$ or $\mathrm{Na}$ in cobalt borophosphates during the HER [32]. However, owing to the poor structural flexibility of many materials, most surface reconstructions are triggered by electro-oxidation during the electrocatalysis process [33], and it is difficult to control the reconstruction during the HER. Furthermore, the evolution of the structural and physicochemical properties of catalysts in the electrocatalysis process makes it difficult to promote HER catalytic performance through electro-oxidation

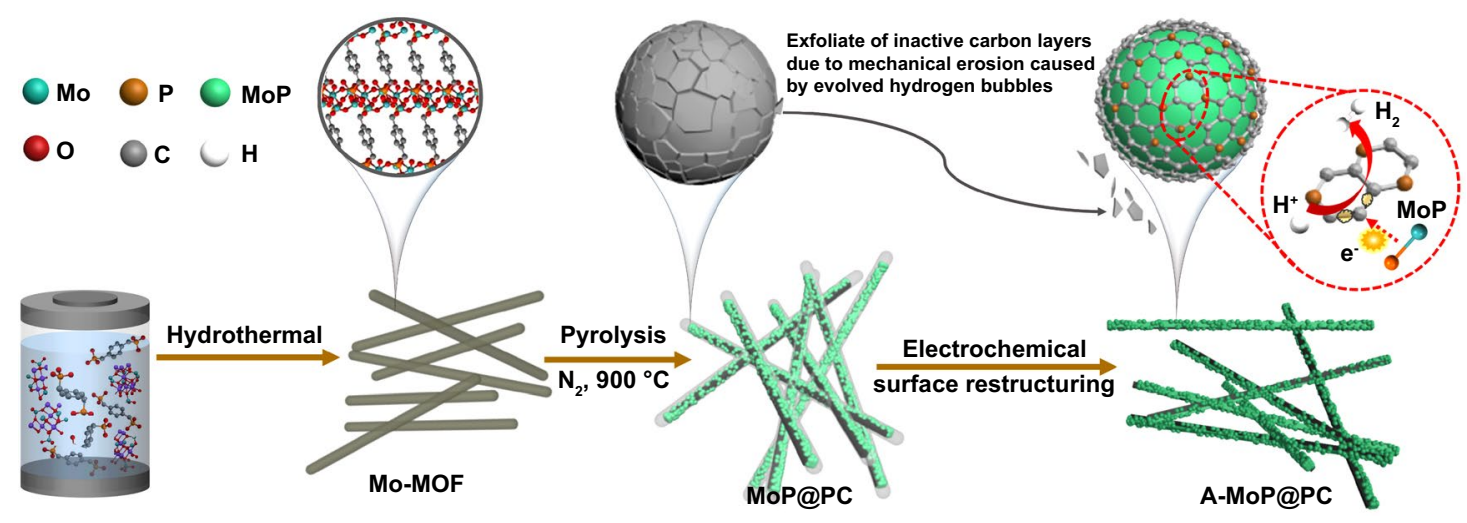

Fig. 1 Schematic illustration for the synthesis of the A-MoP@PC 
methods, especially when surface reconstruction occurs through water electrolysis. This is a significant bottleneck to tracking the structural evolution of catalysts and understanding the nature of catalytically active surfaces [34-36]. Further modulating the surface restructuring process and manipulating the in situ generated active surface species are even more challenging. Therefore, a controllable process is required to adjust the surface carbon structure of electrocatalysts and improve their catalytic properties [37-40].

In this research, we report on the surface restructuring behavior of an electrocatalyst with MoP nanocrystals encapsulated in ultrathin phosphorus-doped carbon layers. A simple electrochemical cycling process is used to peel off the inactive carbon coating layer, which leads to the in situ generated active surface species boosting the HER catalytic activity of the electrocatalysts. The synthesis routine is schematically illustrated in Fig. 1. First, we developed a one-step strategy for the synthesis of a several-layer-thick phosphorus-doped carbon-coated MoP catalyst in a well-defined nanowire structure, derived from a phosphorous-containing Mo-Metal-organic framework (MOF) precursor without any additional phosphorus and carbon sources. Subsequently, the structural evolution of catalysts during a cyclic voltammetry $(\mathrm{CV})$ process was applied to the electrocatalysts. It is important to note that these materials exhibit unusual surface restructuring behavior during the electrochemical process. Using simple low voltage electrochemical cycling in an acidic medium, the inactive surface carbon layers can be exfoliated, which makes the carbon layers thinner, allowing only 1-3 layers to be retained. Because of the high affinity between the MoP core and the P-doped carbon shell, a unique synergistic interaction at the interfaces boosts the electrocatalytic activities of the electrocatalyst. The charge-polarized P-doped carbon layer on the MoP surfaces exhibits excellent stability and catalytic activity. This practical surface restructuring strategy could be utilized to develop efficient electrochemical catalysts that are not only limited to use in the HER procedure.

\section{Experimental Section}

\subsection{Synthesis of p-Xylylenediphosphonic Acid $\left(\mathrm{H}_{4} \mathrm{xdp}\right)$}

The ligand was synthesized by reacting alpha, alpha'-Dibromop-xylene with triethyl phosphite and followed by refluxing the obtained oil with conc. hydrochloric acid according to the literature method [41]. Block colorless crystals were obtained from the water solution by slow evaporation.

\subsection{Synthesis of $\left[\left(\mathrm{MoO}_{2}\right)_{2}(\mathrm{xdp})\left(\mathrm{H}_{2} \mathrm{O}\right)_{2}\right] \cdot 2 \mathrm{H}_{2} \mathrm{O}$}

Mo-MOF precursor was prepared according to previous work [41]. In a typical procedure, $\mathrm{Na}_{2} \mathrm{MoO}_{4} \cdot 2 \mathrm{H}_{2} \mathrm{O}(0.240 \mathrm{~g}$, $1.0 \mathrm{mmol}$ ) was stirred together with p-xylylenediphosphonic acid $\left(\mathrm{H}_{4} \mathrm{xdp}\right)(0.140 \mathrm{~g}, 0.5 \mathrm{mmol})$ in $16 \mathrm{~mL}$ deionized water. The $\mathrm{pH}$ of the solution was adjusted to 1 by dropwise addition of conc. hydrochloric acid. The acidified solution was then placed in a $25 \mathrm{~cm}^{3}$ Ace pressure tube and heated at $120^{\circ} \mathrm{C}$ for $15 \mathrm{~h}$. The resultant white crystalline material was thoroughly washed with deionized water several times and dried at $80^{\circ} \mathrm{C}$ for $12 \mathrm{~h}$ under vacuum.

\subsection{Preparation of MoP@PC Nanowires}

In a typical procedure, $500 \mathrm{mg}$ Mo-MOF precursor was placed in a porcelain boat. Then, the boat was heated at $900{ }^{\circ} \mathrm{C}$ under a constant flow of $\mathrm{N}_{2}$ at $30 \mathrm{~mL} \mathrm{~min}^{-1}$ for 120 min with the warming rate of $20^{\circ} \mathrm{C} \mathrm{min}^{-1}$. The final black powder was collected when the temperature dropped to room temperature under $\mathrm{N}_{2}$.

\subsection{Electrochemical Activation}

The in situ electrochemical activation was carried out in $0.5 \mathrm{M} \mathrm{H}_{2} \mathrm{SO}_{4}$ under a $\mathrm{N}_{2}$ atmosphere to avoid possible oxidation caused by $\mathrm{O}_{2}$ in air. This was conducted by using the three-electrode system of CHI 760E electrochemical workstation (CH Instruments, Inc., Shanghai). MoP@ PC was used as the working electrode, carbon rod was used as the counter electrode, and $\mathrm{Ag} / \mathrm{AgCl}$ (saturated $\mathrm{KCl}$ filled) was used as the reference electrode. The electrochemical activation was performed by cycle voltammetry (CV) from -0.2 to $0.2 \mathrm{~V}$ vs RHE in $0.5 \mathrm{M} \mathrm{H}_{2} \mathrm{SO}_{4}$.

\subsection{Characterization}

The crystal structure of sample was characterized by powder X-ray diffraction (XRD) (PANalytical Inc.) using $\mathrm{Cu}$ 
$\mathrm{K} \alpha$ irradiation operating at $45 \mathrm{kV}$ and $40 \mathrm{~mA}$ with a fixed slit. Morphology of sample was observed by a JEOL JSM7500F (Japan) field-emission scanning electron microscopy (FESEM). High-resolution transmission electron microscopy (HRTEM) images were measured using a JEOL JEM2100F (Japan) transmission electron microscope. Nitrogen sorption isotherms were measured at $77 \mathrm{~K}$ using an Autosorb volumetric gas sorption analyzer (Quantachrome, USA). Thermogravimetric analyzer (TGA) was conducted on Mettler Toledo TGA/SDTA85. X-ray photoelectron spectroscopy
(XPS) analyses were performed with a Thermo ESCALAB 250 (USA) spectrometer using an $\mathrm{Al} \mathrm{K}_{\alpha}(1486.6 \mathrm{eV})$ photon source. Raman spectrum was recorded using JY HR800 under ambient conditions. The X-ray absorption near-edge structure (XANES) measurement was taken at Singapore Synchrotron Light Source, facility for catalysis research (XAFCA) beamline.

Electrochemical measurements were taken at room temperature; catalyst ink was typically made by dispersing $20 \mathrm{mg}$ of catalyst in $2 \mathrm{~mL}$ mixture of alcohol and water
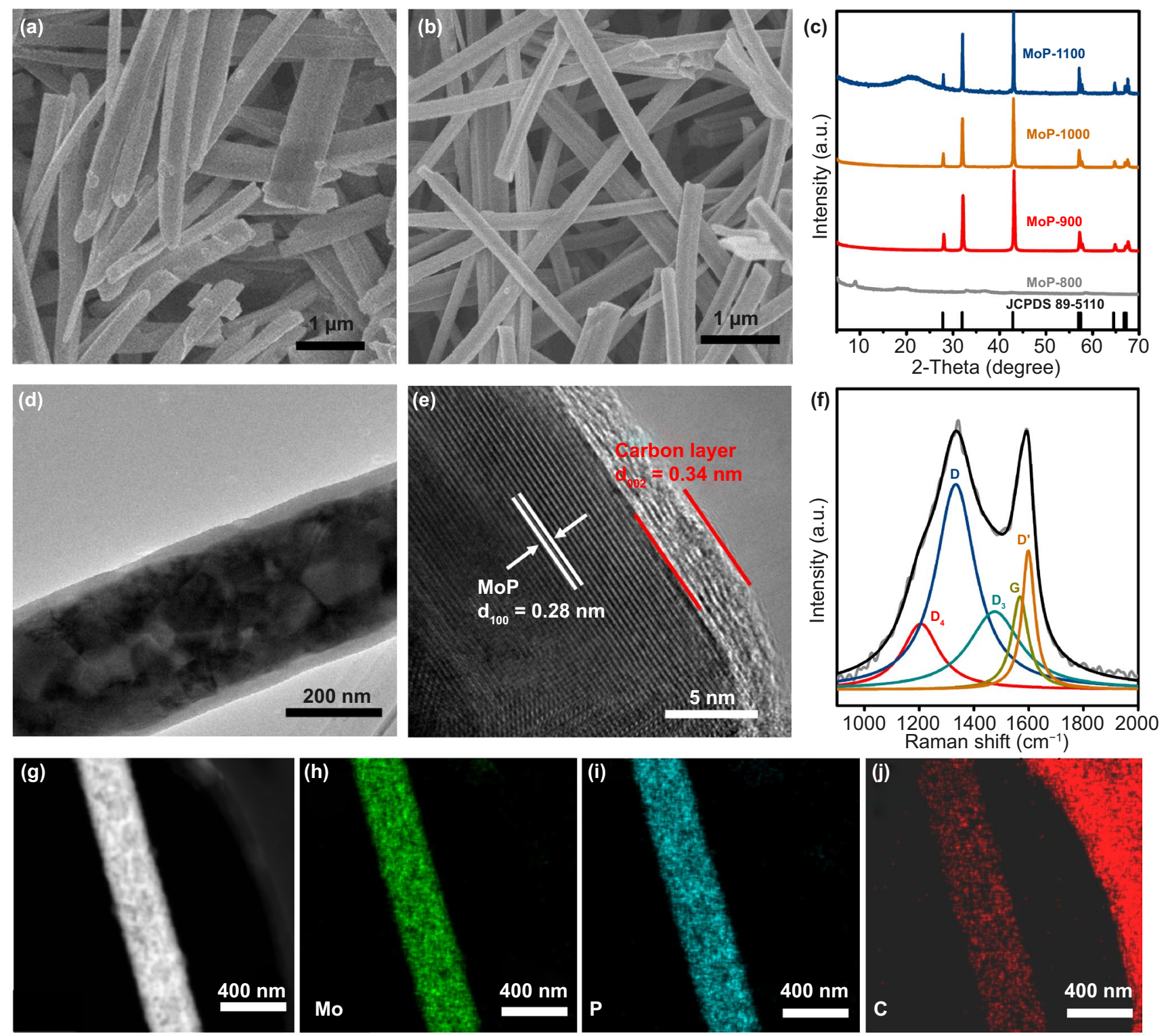

Fig. 2 FESEM images of a Mo-MOF precursor and b MoP@PC. c XRD pattern of Mo-based MOF after being calcined at 700-1100 ${ }^{\circ} \mathrm{C}$. d TEM and e HRTEM images of MoP@PC. f Raman spectra of MoP@PC. g-j TEM-EDS mapping of the MoP@ PC 
(3:1). After adding $0.5 \mathrm{~mL}$ of Nafion solution and ultrasonication, an aliquot of $5 \mu \mathrm{L}$ was pipetted onto the glassy carbon electrode $\left(0.0706 \mathrm{~cm}^{2}\right)$ to reach the catalyst loading of $0.56 \mathrm{mg} \mathrm{cm}^{-2}$. In a three-electrode configuration, polarization curves were collected by $\mathrm{CHI} 760 \mathrm{E}$ electrochemical workstation at room temperature. Current density was normalized to the geometrical area of the working electrode. Polarization data are collected at the scan rate of $5 \mathrm{mV} \mathrm{s}^{-1}$. EISs were carried out in a potentiostatic mode in the frequency range of $10^{6}$ to $1 \mathrm{~Hz}$ with the amplitude of $5 \mathrm{mV}$.

\section{Results and Discussion}

\subsection{Characterization of Morphology and Structure}

The MoP catalysts were synthesized via the one-step sintering of a Mo-based MOF. The XRD peaks of synthesized Mo-MOF agree well with the profile simulated from single-crystal structure data (Fig. S1) [41]. It can be seen in Fig. 2a that the Mo-based MOF has a regular morphology of nanowires, with diameters ranging from approximately 200 to $300 \mathrm{~nm}$. Thermogravimetric (TG) analysis was carried out in $\mathrm{N}_{2}$ atmosphere to characterize the thermostability of the Mo-MOF precursor (Fig. S2). When the temperature is at around $135{ }^{\circ} \mathrm{C}$, the sample lost about $12 \%$ of its weight including water, and small molecules adsorbed from air. The continuous weight-loss in the range of $800-940{ }^{\circ} \mathrm{C}$ corresponds to the decomposition of Mo-MOF precursor. The $\mathrm{N}_{2}$ adsorption/desorption isotherm at $77 \mathrm{~K}$ was performed to investigate specific surface area of Mo-MOF precursor. As shown in Fig. S3, the specific surface area of Mo-MOF precursor is $15.5 \mathrm{~m}^{2} \mathrm{~g}^{-1}$. Subsequently, the Mo-MOF precursor was heat-treated in a nitrogen atmosphere. Figure $2 \mathrm{c}$ shows the XRD pattern of the Mo-based MOF after being calcined at $800-1100{ }^{\circ} \mathrm{C}$. It can be seen that the annealing temperature plays an important role in the fabrication process; the MoP phase was formed when the annealing temperature was higher than $900{ }^{\circ} \mathrm{C}$. The diffraction peaks found through $\mathrm{XRD}$ at $27.8^{\circ}, 31.9^{\circ}, 42.8^{\circ}, 56.9^{\circ}, 57.4^{\circ}, 64.5^{\circ}, 66.8^{\circ}$, and $67.2^{\circ}$ were ascribed to the (001), (100), (101), (110), (002), (111), (200), and (102) planes of hexagonal MoP (JCPDS No. 89-5110), respectively. Furthermore, no other peaks were observed, indicating the good crystallinity and purity of the MOF-derived MoP. The HER activity of these catalysts shown in Fig. S4 helped optimizing the annealing temperatures. The samples obtained at $900{ }^{\circ} \mathrm{C}$ (termed as MoP@PC) possessed better HER activity, which can be seen through the linear sweep voltammetry (LSV) curves in $0.5 \mathrm{M} \mathrm{H}_{2} \mathrm{SO}_{4}$, indicating that the annealing temperature of $900{ }^{\circ} \mathrm{C}$ is optimal for MoP formation and the electrocatalytic activity of MoP@PC. The morphological features of MoP@PC were obtained through field-emission scanning electron microscopy (FESEM) and TEM. The FESEM of MoP@PC (Fig. 2b) shows that the MoP@PC has a regular nanowire morphology, similar to that of the Mo-MOF precursor (Fig. 2a), indicating that the initial morphology of the precursor is maintained well after pyrolysis. The nanowire morphology was also obtained through the TEM images. In Fig. 2d, e, the HRTEM shows that the nanowire structures consist of MoP cores and graphene shells (Fig. 2d). The nitrogen adsorption/desorption isotherm at $77 \mathrm{~K}$ of MoP@ $\mathrm{PC}$ indicating the specific surface area of $11.7 \mathrm{~m}^{2} \mathrm{~g}^{-1}$ (Fig. S5).

The HRTEM image in Fig. 2e clearly shows the crystal planes with a d-spacing of $0.28 \mathrm{~nm}$, corresponding to the (100) lattice of the MoP nanoparticles [42], further confirming that the MoP was synthesized successfully with a high degree of crystallinity. Moreover, the MoP nanoparticles are surrounded by thick graphite shells with an interlayer distance of $0.34 \mathrm{~nm}$, and the carbon shells number at about 10 layers.

To further confirm the chemical compositions of the MoP@PC, Raman spectroscopy was performed (Fig. 2f). The deconvoluted $\mathrm{D}\left(\approx 1323 \mathrm{~cm}^{-1}\right)$ and $\mathrm{D}^{\prime}\left(1600 \mathrm{~cm}^{-1}\right)$ bands originate from defects such as the edge sites and structural disorders in the graphite shell of MoP@PC, while the observed $\mathrm{G}$ band $\left(\approx 1568 \mathrm{~cm}^{-1}\right)$ is assigned to the in-plane stretching between $s p^{2}$-graphitic carbons. The deconvoluted $\mathrm{D}_{4}$ band $\left(\approx 1200 \mathrm{~cm}^{-1}\right)$ indicates the presence of amorphous carbon, while the $\mathrm{D}_{3}$ band $\left(\approx 1490 \mathrm{~cm}^{-1}\right)$ can be ascribed to the disordered graphitic lattice [29]. The low ratio of the $\mathrm{G}$ band demonstrates the existence of a highly disordered graphite structure in the carbon shell of MoP@PC [43]. The uniform distribution without significant aggregations of phosphorus, carbon, and molybdenum is observed through TEM energy-dispersive X-ray (EDX) spectroscopy, as shown in Fig. $2 g-j$. XPS measurements were applied to elucidate the surface chemistry of MoP@PC. The XPS spectra 

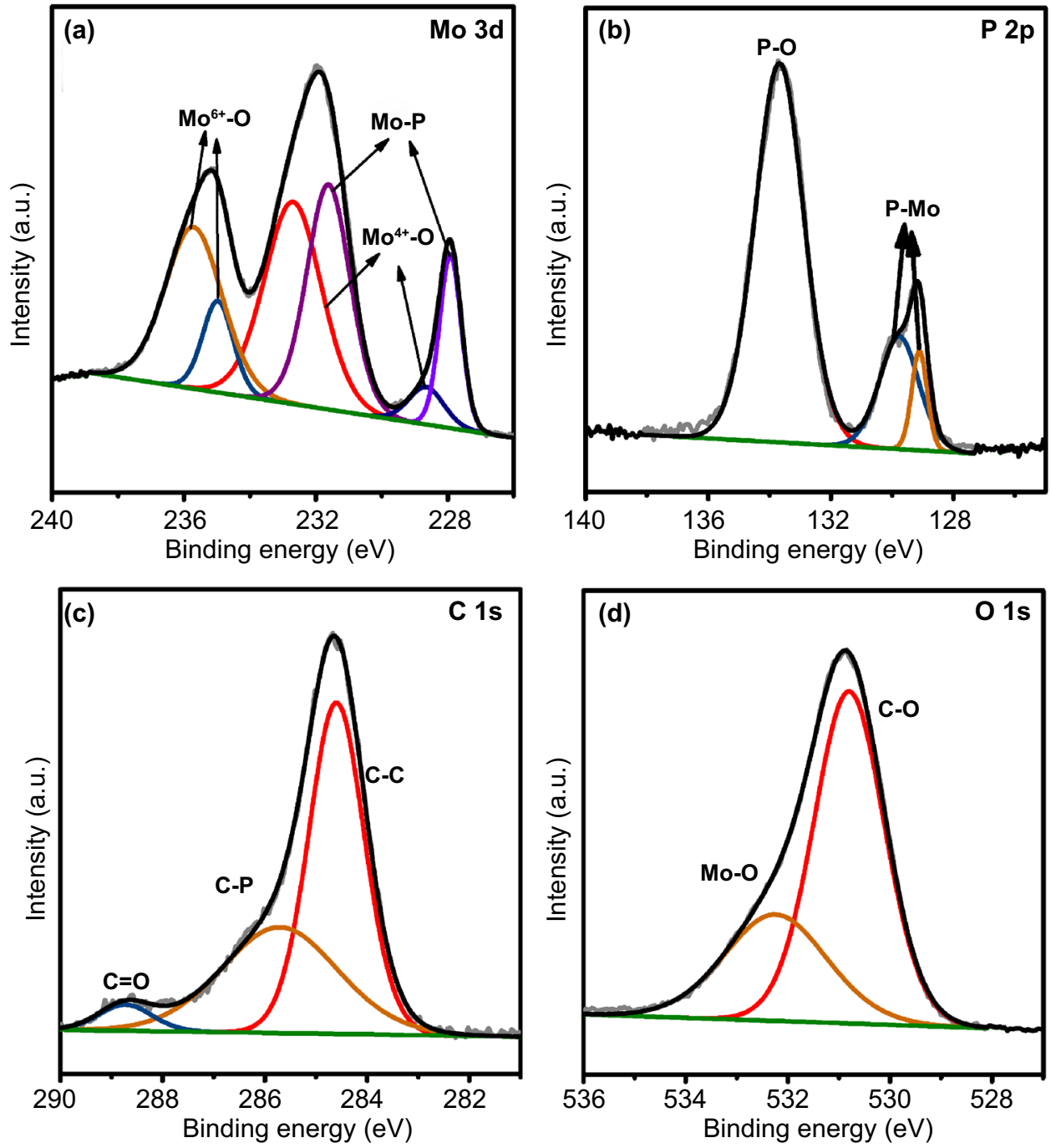

Fig. 3 High-resolution XPS of a Mo 3d, b P 2p, c C 1 s, and d O 1 s of MoP@PC

of MoP@PC indicate the existence of Mo, P, C, and O (Fig. S6). Figure 3a-d shows the typical high-resolution XPS profiles of Mo 3d, P 2p, C 1 s, and O $1 \mathrm{~s}$ in the MoP@PC, respectively. Two peaks (Fig. 3a) at the low binding energies of 228.3 and $231.5 \mathrm{eV}$, are assigned to $\mathrm{Mo}^{3+}$ in $\mathrm{MoP}$. In Fig. 3a, the four peaks at 236.2, $234.4\left(\mathrm{Mo}^{6+} 3 \mathrm{~d}_{3 / 2} / 3 \mathrm{~d}_{5 / 2}\right)$, 233.5, and $229.0\left(\mathrm{Mo}^{4+} 3 \mathrm{~d}_{3 / 2} / 3 \mathrm{~d}_{5 / 2}\right) \mathrm{eV}$ indicate that a small amount of MoP on the surface was oxidized to $\mathrm{MoO}_{3}$ or $\mathrm{MoO}_{2}$ [44]. The content of the molybdenum oxide present on the surface of MoP@PC was quite low, so it could not be detected by XRD. The P 2p peaks (Fig. 3b) at 129.5 and $130.4 \mathrm{eV}$ are attributed to low valence $\mathrm{P}$ in MoP, and the peak at a higher binding energy $(133.8 \mathrm{eV})$ is assigned to the $\mathrm{PO}_{4}{ }^{3-}$ or $\mathrm{P}_{2} \mathrm{O}_{5}$ that arises from surface oxidation of $\mathrm{P}$ compounds and MoP species [45]. The graphitic carbon is found to be the major species, as reflected in the main peak of the $\mathrm{C} 1 \mathrm{~s}$ spectrum, at $284.6 \mathrm{eV}$ (Fig. 3c). The peak around $286.1 \mathrm{eV}$ is attributed to the carbon in the $\mathrm{C}-\mathrm{P}$ bonds [46], which is a product of the phosphorization reaction and carbon in the graphene. The peak around $288.5 \mathrm{eV}$ can be attributed to $\mathrm{C}=\mathrm{O}$. Figure $3 \mathrm{~d}$ shows the XPS spectrum of the $\mathrm{O} 1 \mathrm{~s}$ energy region, which can be deconvoluted into two peaks. The molybdenum oxides caused the peak at $532.2 \mathrm{eV}$, and another peak at $530.2 \mathrm{eV}$ can be attributed to the $\mathrm{C}-\mathrm{O}$ bonds between the oxygen-containing groups and $\mathrm{C}$ atoms. 

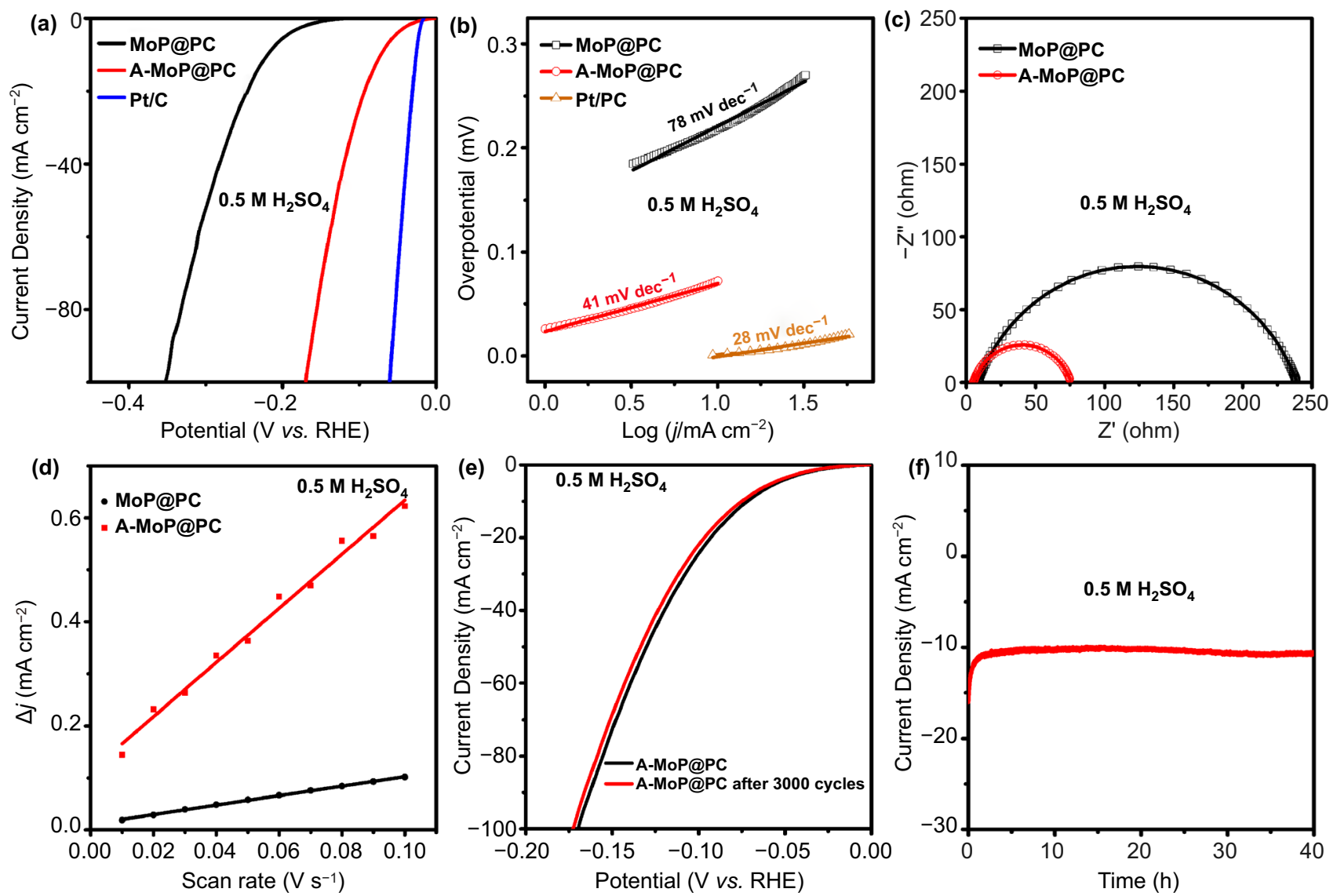

Fig. 4 a LSV curves, b Tafel plots, $\mathbf{c}$ Nyquist plots, and $\mathbf{d}$ plots of the capacitive currents of MoP@ PC and A-MoP@ PC. e and $\mathbf{f}$ Stability test of A-MoP@PC for HER in $0.5 \mathrm{M} \mathrm{H}_{2} \mathrm{SO}_{4}$

These results reveal that the one-step annealing of the MoMOF precursor in a nitrogen atmosphere and appropriate annealing temperatures can encapsulate MoP in a thick carbon layer. During calcination, the Mo and $\mathrm{P}$ in the Mo-MOF precursor form MoP in situ. Simultaneously, the organic moiety in the Mo-MOF structure can provide carbon sources for the in situ generation of a carbon matrix surrounding the MoP. The intrinsic coordination environment of Mo-MOF causes the phosphorus-doped carbon shell to adhere strongly to the MoP core. Taking advantage of the unique metalsupport interactions between the MoP and carbon matrix, enhanced electron transport could be achieved, which facilitates the control of the restructured active surface.

\subsection{Electrocatalytic Measurement}

The electrocatalytic activity of MoP@PC was investigated in an Ar-saturated $0.5 \mathrm{M} \mathrm{H}_{2} \mathrm{SO}_{4}$ solution. To mitigate the influence of platinum, a carbon rod was used as the counter electrode. The HER performance of the commercial $20 \mathrm{wt} \% \mathrm{Pt} / \mathrm{C}$ catalyst was measured for comparison. The LSV curves are shown in Fig. 4a. The black curve shows the initial performance of MoP@PC. Its high overpotential $\left(\eta_{10}=233 \mathrm{mV}\right)$ could be caused by the negative effect of the excessive surface carbon layer shown in TEM results (Fig. 2d, e). Chen et al. [47] eliminated the carbon using oxygen plasma, which requires a plasma generator and does not satisfy the requirements of in situ activation on the electrode. In this study, we found that simple electrochemical activation using $\mathrm{CV}$ (a part of the $\mathrm{CV}$ activated process is shown in Fig. S7) could restructure the surface with significantly improved HER performance. In comparison with other activation methods, this type of electrochemical activation approach is very simple yet effective for constructing active surfaces. As shown in Fig. 4a, the HER performance of MoP@PC after activation for 12 h (termed as A-MoP@ 
PC) is significantly improved. To achieve a current density of $10 \mathrm{~mA} \mathrm{~cm}{ }^{-2}$, the A-MoP@PC only required an overpotential of $68 \mathrm{mV}$, which is much lower than that of freshly synthesized MoP@PC, and better than most reported electrocatalysts (Table S1). The Tafel plots of the electrocatalysts were measured to evaluate the intrinsic HER kinetic process. As shown in Fig. 4b, the Tafel slopes of MoP@P and A-MoP@ PC were 78 and $41 \mathrm{mV} \mathrm{dec}^{-1}$, respectively. The significant decrease in the Tafel slope illustrates that, after $12 \mathrm{~h}$ of $\mathrm{CV}$ activation, the rate-limiting step for the HER of A-MoP@PC
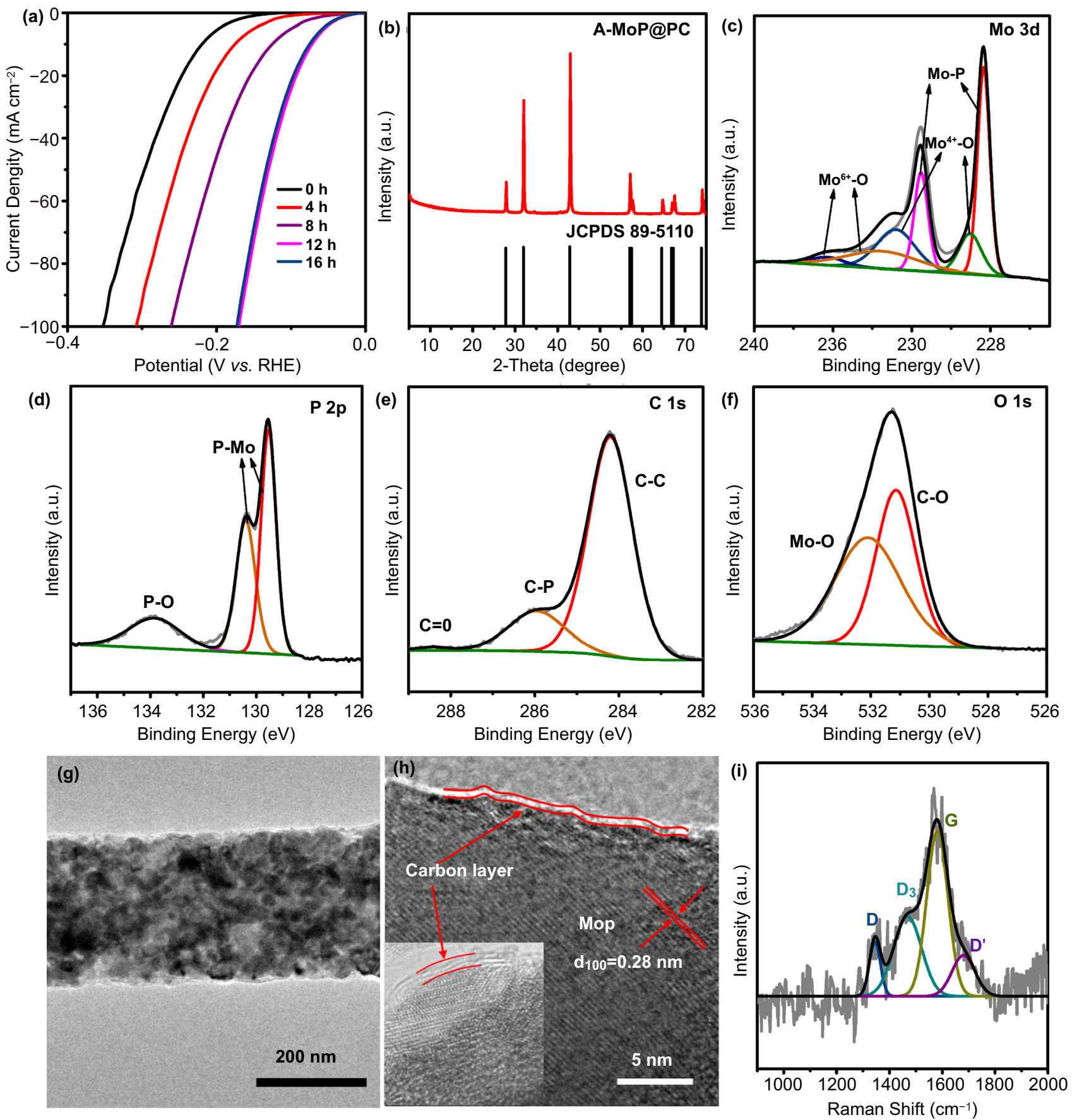

Fig. 5 a HER polarization curves of MoP@PC activation for different times in $0.5 \mathrm{M} \mathrm{H}_{2} \mathrm{SO}_{4}$. b XRD pattern of A-MoP@PC. High-resolution XPS of $\mathbf{c}$ Mo 3d, d P 2p, e C 1 s, and f O 1 s of A-MoP@PC. g TEM and h HRTEM of A-MoP@PC. i Raman spectrum of A-MoP@PC 
changes from the Volmer step to the Heyrovsky step, which indicates that the Volmer step is accelerated significantly and the reaction kinetics are improved.

The performance improvement of A-MoP@PC in terms of the HER became more apparent based on the Nyquist plots (Fig. 4c) obtained using electrochemical impedance spectroscopy (EIS). The charge transfer resistance of MoP@ PC decreased dramatically after $\mathrm{CV}$ activation, suggesting that A-MoP@PC has more facile electrode kinetics. The electrochemical active surface areas (ECSA) of MoP@PC and A-MoP@PC were calculated using the double-layer capacitance $\left(\mathrm{C}_{\mathrm{dl}}\right)$ [48-50], and could be utilized to evaluate the intrinsic activity [51]. The calculated $\mathrm{C}_{\mathrm{dl}}$ values (see more details in the supporting information) of MoP@ PC and A-MoP@PC (Fig. 4d) are 0.9 and $5.2 \mathrm{mF} \mathrm{cm}{ }^{-2}$, respectively. The corresponding ECSA values are $22.9 \mathrm{~cm}^{2}$ for MoP@C and $148.7 \mathrm{~cm}^{2}$ for A-MoP@C. The increased $\mathrm{C}_{\mathrm{dl}}$ and ECSA values of A-MoP@PC demonstrated that the higher electrocatalytic activity after activation was caused by the increases of the restructured active surface. In addition to the aforementioned features, stability is an essential factor affecting the performance of electrocatalysts. The stability of the A-MoP@PC catalyst was evaluated using a CV scan with 3000 cycles. As shown in Fig. 4e, A-MoP@PC had similar polarization curves, demonstrating negligible losses in catalytic activity. The chronoamperometric electrolysis curve for $40 \mathrm{~h}$ in Fig. $4 \mathrm{f}$ also indicates the good durability of A-MoP@PC in acidic electrolytes.

\subsection{Underlying Mechanism}

It is of great research interest to investigate the CV-induced electrochemical activation mechanism. The HER polarization curves of catalysts after $0,4,8,12$, and $16 \mathrm{~h}$ of electrochemical activation $\mathrm{CV}$ cycles are shown in Fig. 5a. The four curves for $0,4,8$, and $12 \mathrm{~h}$ differ significantly, which indicates an improvement in HER electrocatalytic performance, proving that the accessible potent catalytic surfaces increase gradually from 0 to $12 \mathrm{~h}$. After $12 \mathrm{~h}$ of electrochemical activation, the variation trend ceased. The polarization curve of the catalyst after $16 \mathrm{~h}$ of electrochemical activation almost overlapped with that for $12 \mathrm{~h}$ of electrochemical activation, suggesting that the restructuring process was completed and the restructured structure was stable.
To acquire a deeper understanding of the underlying mechanism, A-MoP@PC was investigated using different types of characterization. To understand the morphology and material composition of the A-MoP@PC catalyst, XRD, XPS, BET, TEM, Raman, and X-ray near-edge structure (XANES) characterizations were performed. The XRD patterns in Fig. 5b show that the bulk phase of A-MoP@ $\mathrm{PC}$ remains unchanged after electrochemical activation. Figure $5 \mathrm{c}-\mathrm{f}$ shows the XPS profiles of Mo 3d, P 2p, C $1 \mathrm{~s}$, and O 1 s of A-MoP@PC, respectively. In comparison with the Mo 3d (Fig. 3a) and P 2p (Fig. 3b) profiles of MoP@ PC, the stronger peaks of Mo $3 \mathrm{~d}$ (Fig. 5c) at lower binding energies (227.9 and $231.8 \mathrm{eV}$ ) can be assigned to $\mathrm{Mo}^{3+}$, and the peak located at $129.8 \mathrm{eV}$ of $\mathrm{P} 2 \mathrm{p}$ (Fig. $5 \mathrm{~d}$ ) proves the improved penetration of MoP for A-MoP@PC, confirming that a portion of the surface carbon layer disappeared. As shown in Fig. 3c, d for MoP@PC, no significant change can be seen in terms of $\mathrm{C} 1 \mathrm{~s}$ (Fig. 5e) and $\mathrm{O} 1 \mathrm{~s}$ (Fig. 5f). As shown in Fig. S9, the A-MoP@PC displays similar $\mathrm{N}_{2}$ adsorption/ desorption isotherm with MoP@PC, the specific surface area of A-MoP@PC is $14 \mathrm{~m}^{2} \mathrm{~g}^{-1}$, and there is no significant change in the pore size distribution. The TEM and HRTEM images in Fig. 5g, h, for A-MoP@PC, show that the nanowire structure is maintained well after electrochemical activation, and the surface carbon layers become thinner than the MoP@PC (Fig. 2d, e). Figure 5i shows the Raman spectra of sample A-MoP@PC, which significantly differs from that of MoP@PC, which is shown in Fig. 2f. The disappearance of the $\mathrm{D}_{4}$ band demonstrates the dissolution of the amorphous carbon during electrochemical activation. The observed $\mathrm{G}$ band $\left(\approx 1577 \mathrm{~cm}^{-1}\right)$ confirms the preservation of stable graphitic carbon on the surface. This could be attributed to the fact that the amorphous carbon shell dissolved in the harsh chemical environment during the electrochemical surface restructuring process. Thick graphite exfoliation takes place continuously until it reaches the closest 1-3 layers to the MoP core; subsequently, the modified electronic structure inhibits further exfoliation.

To assess the structural stability of A-MoP@ PC, SEM/ TEM was performed. SEM images of the post-HER sample indicated its well-defined nanowire structures, similar to the A-MoP@PC before the HER stability testing (Fig. S10). TEM and HRTEM images (Fig. S11) of A-MoP@PC after the stability testing clearly show that the regular nanowire 

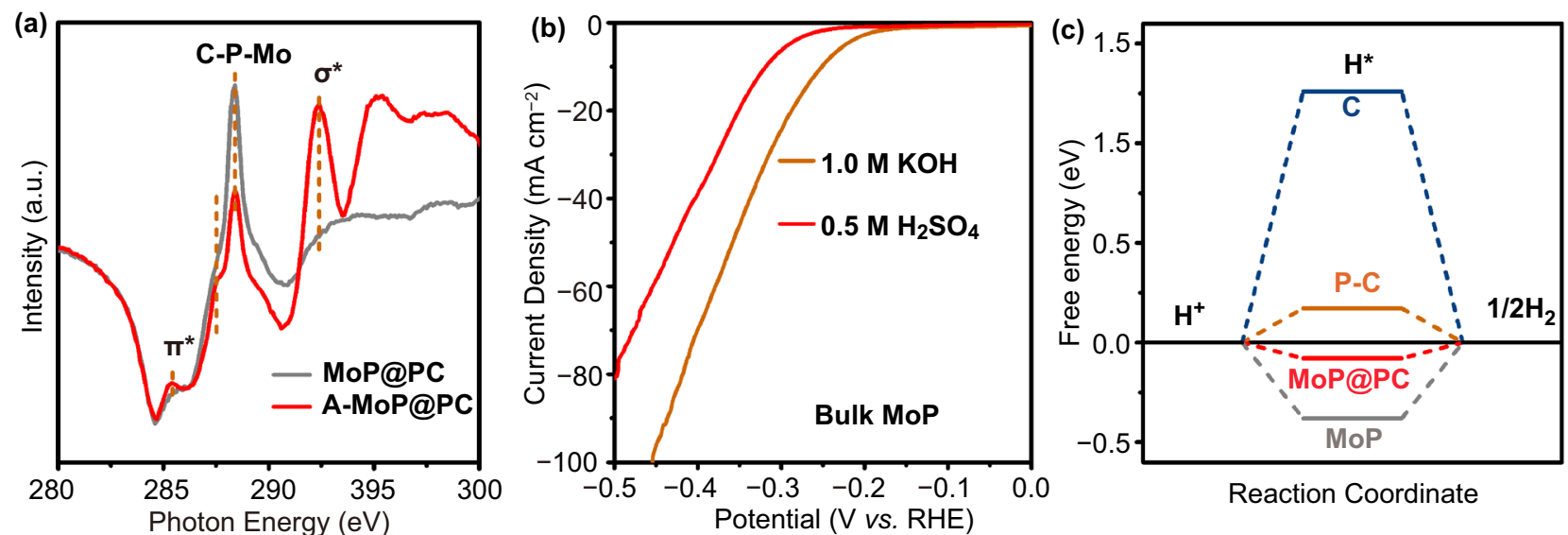

(d)

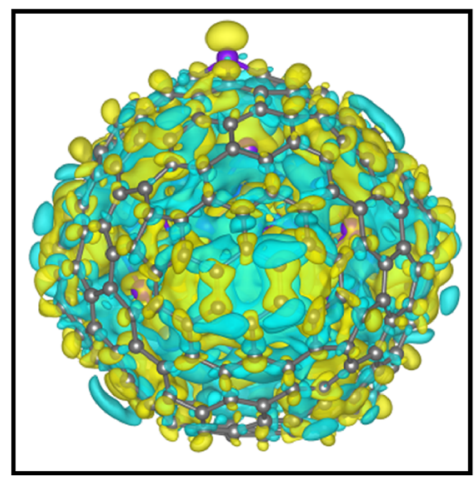

(e)
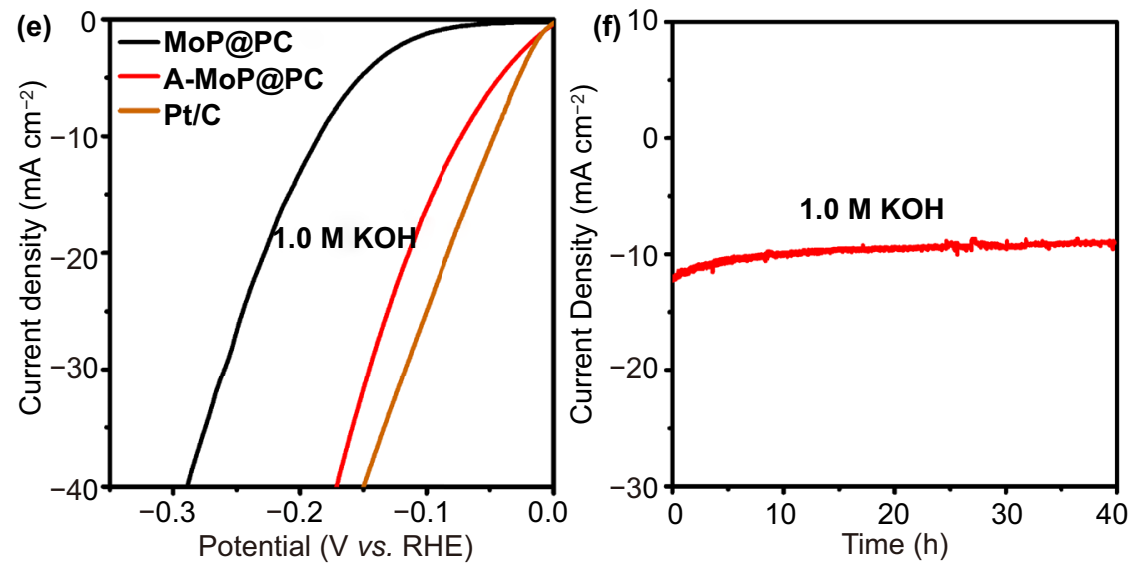
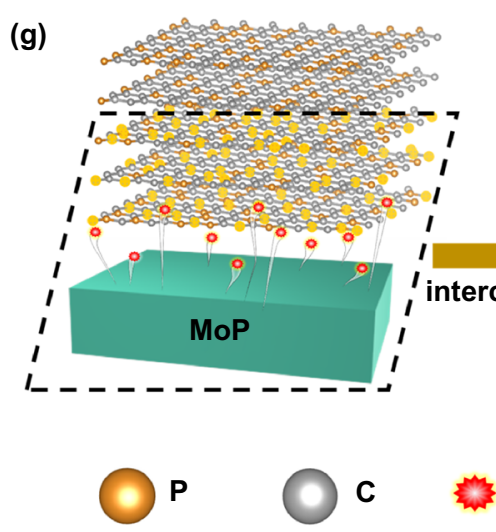

$\mathrm{H}^{+}$

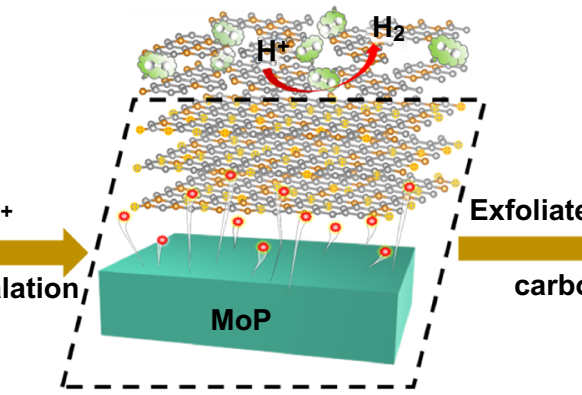

Hydrogen Bubble

Synergistically Interactive Catalytic Active Sites of MoP \& Phosphorus Doped Carbon

Fig. 6 a C K-edge XANES of MoP@PC and A-MoP@PC. b HER polarization curves of commercial MoP in $0.5 \mathrm{M} \mathrm{H}_{2} \mathrm{SO}_{4}$ and $1.0 \mathrm{M} \mathrm{KOH}$. c The calculated free energy diagrams for HER of various catalysts. d Three-dimensional charge-density difference for MoP@PC hybrids with an isovalue of $0.0006 \mathrm{e}^{-3}$. Yellow and glaucous isosurfaces represent charge accumulation and depletion in the space with respect to isolated MoP clusters and phosphorus-doped carbon shell. e HER polarization curves of MoP@PC and A-MoP@PC in 1.0 M KOH. f Stability test of A-MoP@PC in $1.0 \mathrm{M} \mathrm{KOH}$. $\mathrm{g}$ Schematic representation for the restructuring process and mechanism

was well covered by ultrathin carbon layers, demonstrating that the A-MoP@PC still well maintained its original features after the long-term stability test. Surface composition and elements valences of the A-MoP@PC after stability were evaluated by XPS. Results are shown in Fig. S12 with a good preservation of the Mo 3d, P 2p, C $1 \mathrm{~s}$, and O $1 \mathrm{~s}$ 
binding energies. High-resolution XPS spectra for Mo 3d (Fig. S12a), P 2p (Fig. S12b), C 1 s (Fig. S12c), and O $1 \mathrm{~s}$ (Fig. S12d) confirmed negligible differences between the original and post-HER samples, suggesting that the sample properly retained its chemical composition and structure after long-term amperometry process.

XANES measurements were carried out to investigate the interaction between the MoP nanoparticles and phosphorusdoped carbon layers. Figure 6a shows the C K-edge XANES spectra of MoP@PC and A-MoP@PC. The absorption features at 285.4 and $292.4 \mathrm{eV}$ can be assigned to the $\mathrm{C} 1 \mathrm{~s}$ transitions of the graphitic $\mathrm{C}-\mathrm{C} \pi^{*}$ and $\mathrm{C}-\mathrm{C} \delta^{*}$ states, respectively [52]. For A-MoP@PC, the intensity of the peaks for $\pi^{*}$ and $\delta^{*}$, increased significantly, indicating that the graphitization degree of A-MoP@ PC is improved, which can be attributed to the dissolution of the amorphous carbon; this can be also observed in the aforementioned Raman spectra. There are two peaks between the peak for $\pi^{*}$ and $\delta^{*}$. The peak located at about 288 eV of A-MoP@PC can be attributed to a graphene analog of the interlayer state in graphite, which indicates the thinning of the thick carbon shell and the creation of the ultrathin graphene layers [53]. The peak at 288.4 eV can be ascribed to Mo-P-C bonds [54-57], suggesting a significant chemical interaction between the MoP and phosphorus-doped carbon layers. The intensity for the Mo-P-C peak of A-MoP@ PC is slightly weaker than that of MoP@PC, which could be attributed to the elimination of the superfluous carbon and the thinning of the phosphorus-doped carbon shell during the electrochemical surface restructuring process. This result is also consistent with the measurements discussed above.

To further understand the activation process and identify the nature of the active sites of the catalysts, we investigated the impact of thiocyanate ions $\left(\mathrm{SCN}^{-}\right)$on the HER activity (Fig. S13). $\mathrm{SCN}^{-}$ions can poison the active metal centers because of their strong binding ability with metal atoms [57]. After the addition of $\mathrm{SCN}^{-}$ions, the Mo active sites of MoP@PC were blocked. As shown in Fig. S13, the polarization curve of the MoP@PC electrocatalyst in the $0.5 \mathrm{M}$ $\mathrm{H}_{2} \mathrm{SO}_{4}$ electrolyte exhibits a marked cathodic shift after poisoning by $\mathrm{KSCN}(5 \mathrm{mM})$, implying that the synergistic interaction between MoP and the phosphorus-doped graphene plays a critical role in the catalytic process. Furthermore, MoP also has a certain catalytic effect. To understand the influence of $\mathrm{MoP}$ on the catalytic process, the catalytic activity and stability of commercial MoP were further confirmed for comparison. As shown in Fig. 6b, the commercial MoP needed an overpotential of $319 \mathrm{mV}$ to achieve the current density of $10 \mathrm{~mA} \mathrm{~cm}{ }^{-2}$ in $0.5 \mathrm{M} \mathrm{H}_{2} \mathrm{SO}_{4}$. As shown in Fig. $\mathrm{S} 14$, when estimated by galvanostatic measurement at a constant current of $10 \mathrm{~mA} \mathrm{~cm}^{-2}$ in $0.5 \mathrm{M} \mathrm{H}_{2} \mathrm{SO}_{4}$, obvious performance attenuation was observed within $10 \mathrm{~h}$, the current density fallen $\sim 24 \%$ over $15 \mathrm{~h}$. The unsatisfactory activity and poor stability of commercial MoP further highlight the significant synergistic interactions between the phosphorusdoped carbon and MoP.

DFT simulations using the Vienna Ab initio Simulation Package (VASP) of Mede A software were performed to understand the fundamental reaction mechanism of the HER catalytic process, the relevant theoretical models for which are shown in Figs. S15-S18. It has been proven that the adsorption free energy of $\mathrm{H}\left(\Delta G H^{*}\right)$ is an appropriate parameter for use in evaluating the HER activity of a catalyst, and a catalyst that gives $\Delta G H^{*} \approx 0$ is considered to be a promising candidate for the HER, ensuring rapid proton/electron transfer and a quick hydrogen-release process. As shown in Fig. 6c, the PC exhibits a $\Delta G H^{*}$ value of $0.1716 \mathrm{eV}$, which is much smaller than the value for a carbon shell $(1.259 \mathrm{eV})$, indicating that introducing the $\mathrm{P}$ dopant in the carbon layers can enhance the HER activity. Furthermore, the $\Delta G H^{*}$ of MoP@PC (-0.079 eV) was comparatively higher than that of the pristine MoP surface $(-0.38 \mathrm{eV})$ and $\mathrm{PC}$, which suggests that the phosphorous dopants and enclosed MoP nanocrystals can synergistically promote the HER performance of graphene shells. In summary, the high HER activity of MoP@PC is attributed to the synergy between the MoP and ultrathin P-doped carbon layer. This is in good agreement with the experimental results. The charge-density difference of model MoP@PC was also calculated and is shown in Fig. 6d. It can be seen that the electrons transferred from the MoP core to the P-doped carbon shell, increasing the electron density in the carbon shell; the synergistic effect between MoP and P-doped carbon can reduce the $\Delta G H^{*}$ values to promote the adsorption of $\mathrm{H}^{*}$, enhancing the electrocatalytic performance of the HER.

A schematic of the restructuring process and its underlying mechanism is shown in Fig. 6g. It can be seen that the intrinsic coordination environment of the MOF precursor causes the phosphorus-doped carbon shell of the MoP@PC to adhere strongly to the MoP core. Owing to the strong electronic liberation ability, the MoP core with sufficient electrons can serve as electron donors. The electrons shuttle 
across the phosphorus-doped graphene layers in proximity (1-3 layers) on the MoP core, modifying the surface electronic structures and charge distributions [58], changing the electronic properties of the inert phosphorus-doped graphene layer significantly. This results in alterations to the binding energies of the reaction intermediates on the phosphorus-doped graphene surface, enhancing the electrocatalytic activity. Subsequently, the superfluous inert carbon shell could gradually be stripped off during the $\mathrm{CV}$ process owing to mechanical erosion caused by the evolved hydrogen bubbles; therefore, only the highly active graphene surface remained. Because the modified electronic properties by the MoP core could strengthen the adherence of phosphorusdoped graphene shells, the shutting electrons allow the MoP to adhere strongly to the thin carbon layer; therefore, the binding force between the polarized phosphorus-doped graphene shell and MoP core is strengthened. Further, when the negatively charged carbon layers were modified by MoP, the HER was more likely to take place on the outmost surface, where the reaction molecules and media would be triggered to decompose as soon as they contacted the external highactivity graphene surface. The surface species do not change any more, which indicated that the further surface reconstruction terminated. The phosphorus-doped graphene shells can stabilize the MoP cores under harsh conditions, and the redistributed electrons at the interface of the MoP cores and surface carbon layers enhance the HER activity. Therefore, the catalytic activity of A-MoP@PC is significantly higher than that of any single component. The positive perturbation of the electron distribution endows the outermost surface with excellent catalytic activity, further catalyzing the HER. The synergistic interaction significantly boosts the electrocatalytic activity and stability.

To extend the applicability of this method, the HER performance of A-MoP@PC was investigated in an alkaline electrolyte (1.0 M KOH); a commercial Pt/C sample was evaluated for comparison. Figure 6e shows the LSV curves of MoP@PC and A-MoP@PC catalysts in a 1.0 M KOH solution. The A-MoP@PC exhibited a small overpotential, of $67 \mathrm{mV}$ for $\eta_{10}$, which is better than most reported nonprecious electrocatalysts under alkaline conditions. The corresponding Tafel plots are shown in Fig. S19. The Tafel slopes of MoP@PC and A-MoP@PC are 99 and $40 \mathrm{mV} \mathrm{dec}{ }^{-1}$, respectively, exhibiting more efficient HER kinetics for an A-MoP@ PC catalyst with the Heyrovsky mechanism. The catalytic activity and stability of commercial MoP in $1.0 \mathrm{M}$
$\mathrm{KOH}$ were performed as contrasts. The overpotential was as high as $252 \mathrm{mV}$ to obtain the current density of $10 \mathrm{~mA} \mathrm{~cm}^{-2}$ (Fig. 6b). Meanwhile, the stability of commercial MoP was also poor, the current density decayed rapidly as time progressed (Fig. S14b). The excellent HER catalytic activity of A-MoP@PC is close to that of the Pt/C catalyst and better than most non-noble-metal-based catalysts (Table S1). The chronoamperometric electrolysis curve in Fig. 6 f also exhibits the high durability of A-MoP@PC in alkaline electrolytes; the current density shows ignored decline within $40 \mathrm{~h}$.

\section{Conclusions}

Herein, a new class of phosphorus-doped carbon-coated molybdenum phosphide (A-MoP@PC) electrocatalysts were synthesized through the pyrolysis of a Mo-MOF precursor, followed by a simple electrochemical cycling treatment. The in situ transformation of metal nodes/organic ligands in MOF led to the highly dispersed MoP being protected by phosphorus-doped carbon. During the electrochemical process, the electrons of the inner MoP flow through the carbon layers to the outer surface owing to the greater electron-escape capability, extending the range of MoP in terms of adhering to the thin carbon layer. As a result, the superfluous inactive carbon shell can be stripped off gradually by the $\mathrm{CV}$ process, leading to the restructuring of the electrocatalysts when the active surface carbon was exposed. The activated carbon surfaces not only enhance the catalytic activity but also simultaneously strengthen the durability of the catalyst. DFT calculations indicate that the superior HER performance is a result of the modulation of the electron density and the electronic potential distribution at the graphene surface caused by penetrating electrons from the MoP core. The encapsulated MoP cores act like hearts pumping out electrons into the carbon shell; the electron-rich characteristics of the polarized carbon layer contribute to an increase in the active sites and significantly reduce $\Delta G H^{*}$. These advantages of the electronic modulated structure provide the low overpotentials of $68 \mathrm{mV}$ and $67 \mathrm{mV}$ for the HER, allowing it to reach a current density of $10 \mathrm{~mA} \mathrm{~cm}^{-2}$ in $0.5 \mathrm{M} \mathrm{H}_{2} \mathrm{SO}_{4}$ and $1.0 \mathrm{M} \mathrm{KOH}$, respectively. This strategy can help design superior HER electrocatalysts, providing a promising approach for developing durable electrochemical catalysts. 
Acknowledgements This work was supported by the National Natural Science Foundation of China (Grant Nos. 21975286 and 21473254), the Special Project Fund of "Taishan Scholar" of Shandong Province (Grant No. ts201511017), the QLUT Special Funding for Distinguished Scholars (Grant No. 2419010420), the project ZR2020QE058 supported by Shandong Provincial Natural Science Foundation, the Fundamental Research Funds for the Central Universities (Grant Nos. YCX2020050, 18CX06030A, and 17CX02039A).

Open Access This article is licensed under a Creative Commons Attribution 4.0 International License, which permits use, sharing, adaptation, distribution and reproduction in any medium or format, as long as you give appropriate credit to the original author(s) and the source, provide a link to the Creative Commons licence, and indicate if changes were made. The images or other third party material in this article are included in the article's Creative Commons licence, unless indicated otherwise in a credit line to the material. If material is not included in the article's Creative Commons licence and your intended use is not permitted by statutory regulation or exceeds the permitted use, you will need to obtain permission directly from the copyright holder. To view a copy of this licence, visit http://creativecommons.org/licenses/by/4.0/.

Supplementary Information The online version contains supplementary material available at https://doi.org/10.1007/ s40820-021-00737-w.

\section{References}

1. J. Park, T. Kwon, J. Kim, H. Jin, H.Y. Kim et al., Hollow nanoparticles as emerging electrocatalysts for renewable energy conversion reactions. Chem. Soc. Rev. 47(22), 8173-8202 (2018). https://doi.org/10.1039/c8cs00336j

2. G. Chang, Y. Zhao, L. Dong, D.P. Wilkinson, L. Zhang et al., A review of phosphorus and phosphides as anode materials for advanced sodium-ion batteries. J. Mater. Chem. A 8(10), 4996-5048 (2020). https://doi.org/10.1039/c9ta12169b

3. M. Xiao, L. Zhang, B. Luo, M. Lyu, Z. Wang et al., Moltensalt-mediated synthesis of an atomic nickel co-catalyst on $\mathrm{TiO}_{2}$ for improved photocatalytic $\mathrm{H}_{2}$ evolution. Angew. Chem. Int. Ed. 59(18), 7230-7234 (2020). https://doi.org/10.1002/ anie. 202001148

4. P. Bhanja, Y. Kim, K. Kani, B. Paul, T. Debnath et al., Novel porous metal phosphonates as efficient electrocatalysts for the oxygen evolution reaction. Chem. Eng. J. 396, 125245 (2020). https://doi.org/10.1016/j.cej.2020.125245

5. T. Liu, P. Li, N. Yao, G. Cheng, S. Chen et al., CoP-doped MOF-based electrocatalyst for $\mathrm{pH}$-universal hydrogen evolution reaction. Angew. Chem. Int. Ed. 58(14), 4679-4684 (2019). https://doi.org/10.1002/anie.201901409

6. L. Yan, Y. Xu, P. Chen, S. Zhang, H. Jiang et al., A freestanding 3D heterostructure film stitched by MOF-derived carbon nanotube microsphere superstructure and reduced graphene oxide sheets: a superior multifunctional electrode for overall water splitting and $\mathrm{Zn}$-air batteries. Adv. Mater. 32(48), 2003313 (2020). https://doi.org/10.1002/adma.20200 3313

7. P. Cheng, M. Kim, H. Lim, J. Lin, N.L. Torad et al., A general approach to shaped MOF-containing aerogels toward practical water treatment application. Adv. Sustain. Syst. 4(8), 2000060 (2020). https://doi.org/10.1002/adsu.202000060

8. J. Hou, Z. Wang, P. Chen, V. Chen, A.K. Cheetham et al., Intermarriage of halide perovskites and metal-organic framework crystals. Angew. Chem. Int. Ed. 59(44), 19434-19449 (2020). https://doi.org/10.1002/anie.202006956

9. M.R. Liu, Q.L. Hong, Q.H. Li, Y. Du, H.X. Zhang et al., Cobalt boron imidazolate framework derived cobalt nanoparticles encapsulated in $\mathrm{B} / \mathrm{N}$ codoped nanocarbon as efficient bifunctional electrocatalysts for overall water splitting. Adv. Funct. Mater. 28(26), 1801136 (2018). https://doi.org/10.1002/ adfm.201801136

10. L. Yan, H. Jiang, Y. Xing, Y. Wang, D. Liu et al., Nickel metalorganic framework implanted on graphene and incubated to be ultrasmall nickel phosphide nanocrystals acts as a highly efficient water splitting electrocatalyst. J. Mater. Chem. A 6(4), 1682-1691 (2018). https://doi.org/10.1039/C7TA10218F

11. P. Chen, T. Zhou, M. Zhang, Y. Tong, C. Zhong et al., 3D nitrogen-anion-decorated nickel sulfides for highly efficient overall water splitting. Adv. Mater. 29(30), 1701584 (2017). https://doi.org/10.1002/adma.201701584

12. K. Yao, Z. Xu, M. Ma, J. Li, F. Lu et al., Densified metallic $\mathrm{MoS}_{2}$ /graphene enabling fast potassium-ion storage with superior gravimetric and volumetric capacities. Adv. Funct. Mater. 30(24), 2001484 (2020). https://doi.org/10.1002/adfm.20200 1484

13. Z. Huang, S. Yuan, T. Zhang, B. Cai, B. Xu et al., Selective selenization of mixed-linker Ni-MOFs: $\mathrm{NiSe}_{2} @ \mathrm{NC}$ core-shell nano-octahedrons with tunable interfacial electronic structure for hydrogen evolution reaction. Appl. Catal. B 272, 118976 (2020). https://doi.org/10.1016/j.apcatb.2020.118976

14. X. Wang, Y. Chen, B. Yu, Z. Wang, H. Wang et al., Hierarchically porous $\mathrm{W}$-doped CoP nanoflake arrays as highly efficient and stable electrocatalyst for $\mathrm{pH}$-universal hydrogen evolution. Small 15(37), 1902613 (2019). https://doi.org/10.1002/smll. 201902613

15. J. Hou, Y. Wu, B. Zhang, S. Cao, Z. Li et al., Rational design of nanoarray architectures for electrocatalytic water splitting. Adv. Funct. Mater. 29(20), 1808367 (2019). https://doi.org/ 10.1002/adfm.201808367

16. S. Zhang, W. Xia, Q. Yang, Y.V. Kaneti, X. Xu et al., Coreshell motif construction: highly graphitic nitrogen-doped porous carbon electrocatalysts using MOF-derived carbon@ COF heterostructures as sacrificial templates. Chem. Eng. J. 396, 125154 (2020). https://doi.org/10.1016/j.cej.2020.125154

17. R. Fernandes, A. Chunduri, S. Gupta, R. Kadrekar, A. Arya et al., Exploring the hydrogen evolution capabilities of earthabundant ternary metal borides for neutral and alkaline watersplitting. Electrochim. Acta 354, 136738 (2020). https://doi. org/10.1016/j.electacta.2020.136738 
18. R.K. Hona, S.B. Karki, F. Ramezanipour, Oxide electrocatalysts based on earth-abundant metals for both hydrogen- and oxygen-evolution reactions. ACS Sustain. Chem. Eng. 8(31), 11549-11557 (2020). https://doi.org/10.1021/acssuschemeng. 0c02498

19. L. Li, J. He, Y. Wang, X. Lv, X. Gu et al., Metal-organic frameworks: a promising platform for constructing non-noble electrocatalysts for the oxygen-reduction reaction. J. Mater. Chem. A 7(5), 1964-1988 (2019). https://doi.org/10.1039/ c8ta11704g

20. X. Wang, L. Chai, J. Ding, L. Zhong, Y. Du et al., Chemical and morphological transformation of MOF-derived bimetallic phosphide for efficient oxygen evolution. Nano Energy 62 , 745-753 (2019). https://doi.org/10.1016/j.nanoen.2019.06.002

21. H. Tan, Y. Zhao, W. Xia, J. Zhao, X. Xu et al., Phosphorus- and nitrogen-doped carbon nanosheets constructed with monolayered mesoporous architectures. Chem. Mater. 32(10), 4248-4256 (2020). https://doi.org/10.1021/acs.chemmater. $0 \mathrm{c} 00731$

22. Y.V. Kaneti, Y. Guo, N.L.W. Septiani, M. Iqbal, X. Jiang et al., Self-templated fabrication of hierarchical hollow manganesecobalt phosphide yolk-shell spheres for enhanced oxygen evolution reaction. Chem. Eng. J. 405, 126580 (2021). https://doi. org/10.1016/j.cej.2020.126580

23. F. Li, G.F. Han, H.J. Noh, Y. Lu, J. Xu et al., Construction of porous $\mathrm{Mo}_{3} \mathrm{P} / \mathrm{Mo}$ nanobelts as catalysts for efficient water splitting. Angew. Chem. Int. Ed. 57(43), 14139-14143 (2018). https://doi.org/10.1002/anie.201808844

24. B. Liu, H. Li, B. Cao, J. Jiang, R. Gao et al., Few layered N, P dual-doped carbon-encapsulated ultrafine MoP nanocrystal/ MoP cluster hybrids on carbon cloth: an ultrahigh active and durable 3D self-supported integrated electrode for hydrogen evolution reaction in a wide $\mathrm{pH}$ range. Adv. Funct. Mater. 28(30), 1801527 (2018). https://doi.org/10.1002/adfm.20180 1527

25. W. Xiao, P. Liu, J. Zhang, W. Song, Y.P. Feng et al., Dualfunctional $\mathrm{N}$ dopants in edges and basal plane of $\mathrm{MoS}_{2}$ nanosheets toward efficient and durable hydrogen evolution. Adv. Energy Mater. 7(7), 1602086 (2017). https://doi.org/10. 1002/aenm.201602086

26. P. Xiao, M.A. Sk, L. Thia, X. Ge, R.J. Lim et al., Molybdenum phosphide as an efficient electrocatalyst for the hydrogen evolution reaction. Energy Environ. Sci. 7(8), 2624-2629 (2014). https://doi.org/10.1039/c4ee00957f

27. L. Yu, D. Deng, X. Bao, Chain mail for catalysts. Angew. Chem. Int. Ed. 59(36), 15294-15297 (2020). https://doi.org/ 10.1002/anie.202007604

28. H. Jin, X. Wang, C. Tang, A. Vasileff, L. Li et al., Stable and highly efficient hydrogen evolution from seawater enabled by an unsaturated nickel surface nitride. Adv. Mater. 33(13), 2007508 (2021). https://doi.org/10.1002/adma.202007508

29. J. Deng, P. Ren, D. Deng, X. Bao, Enhanced electron penetration through an ultrathin graphene layer for highly efficient catalysis of the hydrogen evolution reaction. Angew. Chem. Int. Ed. 54(7), 2100-2104 (2015). https://doi.org/10.1002/ anie. 201409524
30. X. Liu, J. Meng, J. Zhu, M. Huang, B. Wen et al., Comprehensive understandings into complete reconstruction of precatalysts: synthesis, applications, and characterizations. Adv. Mater. 33(32), 2007344 (2021). https://doi.org/10.1002/adma. 202007344

31. X. Li, L. Xiao, L. Zhou, Q. Xu, J. Weng et al., Adaptive bifunctional electrocatalyst of amorphous CoFe oxide @ 2D black phosphorus for overall water splitting. Angew. Chem. Int. Ed. 59(47), 21106-21113 (2020). https://doi.org/10.1002/ anie. 202008514

32. P.W. Menezes, A. Indra, I. Zaharieva, C. Walter, S. Loos et al., Helical cobalt borophosphates to master durable overall water-splitting. Energy Environ. Sci. 12(3), 988-999 (2019). https://doi.org/10.1039/c8ee01669k

33. Y. Wang, Y. Zhu, S. Zhao, S. She, F. Zhang et al., Anion etching for accessing rapid and deep self-reconstruction of precatalysts for water oxidation. Matter 3, 2124-2137 (2020). https://doi.org/10.1016/j.matt.2020.09.016

34. X. Wang, C. Xu, M. Jaroniec, Y. Zheng, S.Z. Qiao, Anomalous hydrogen evolution behavior in high-pH environment induced by locally generated hydronium ions. Nat. Commun. 10, 4876 (2019). https://doi.org/10.1038/ s41467-019-12773-7

35. Y. Guo, X. Zhou, J. Tang, S. Tanaka, Y.V. Kaneti et al., Multiscale structural optimization: highly efficient hollow irondoped metal sulfide heterostructures as bifunctional electrocatalysts for water splitting. Nano Energy 75, 104913 (2020). https://doi.org/10.1016/j.nanoen.2020.104913

36. J. Duan, S. Chen, C.A. Ortiz-Ledon, M. Jaroniec, S.Z. Qiao, Phosphorus vacancies that boost electrocatalytic hydrogen evolution by two orders of magnitude. Angew. Chem. Int. Ed. 59(21), 8181-8186 (2020). https://doi.org/10.1002/anie.20191 4967

37. L. Yan, L. Cao, P. Dai, X. Gu, D. Liu et al., Metal-organic frameworks derived nanotube of nickel-cobalt bimetal phosphides as highly efficient electrocatalysts for overall water splitting. Adv. Funct. Mater. 27(40), 1703455 (2017). https:// doi.org/10.1002/adfm.201703455

38. P. Chen, H. Ren, L. Yan, J. Shen, T. Wang et al., Metal-organic frameworks enabled high-performance separators for safetyreinforced lithium ion battery. ACS Sustain. Chem. Eng. 7(19), 16612-16619 (2019). https://doi.org/10.1021/acssu schemeng.9b03854

39. C.C. Hou, H.F. Wang, C. Li, Q. Xu, From metal-organic frameworks to single/dual-atom and cluster metal catalysts for energy applications. Energy Environ. Sci. 13(6), 1658-1693 (2020). https://doi.org/10.1039/c9ee04040d

40. Y. Wang, Y.Y. Xue, L.T. Yan, H.P. Li, Y.P. Li et al., Multimetal incorporation into 2D conductive metal-organic framework nanowires enabling excellent electrocatalytic oxidation of benzylamine to benzonitrile. ACS Appl. Mater. Interfaces 12(22), 24786-24795 (2020). https://doi.org/10.1021/acsami.0c05094

41. A.A. Ayi, A.D. Burrows, M.F. Mahon, V.M. Sebestyen, A molybdenum diphosphonate network structure exhibiting reversible dehydration and selective uptake of methanol. 
CrystEngComm 15(45), 9301-9303 (2013). https://doi.org/ 10.1039/c3ce40484f

42. K. Liang, S. Pakhira, Z. Yang, A. Nijamudheen, L. Ju et al., S-doped MoP nanoporous layer toward high-efficiency hydrogen evolution in pH-universal electrolyte. ACS Catal. 9(1), 651-659 (2018). https://doi.org/10.1021/acscatal.8b04291

43. L. Yan, H. Jiang, Y. Wang, L. Li, X. Gu et al., One-step and scalable synthesis of $\mathrm{Ni}_{2} \mathrm{P}$ nanocrystals encapsulated in $\mathrm{N}$, $\mathrm{P}$-codoped hierarchically porous carbon matrix using a bipyridine and phosphonate linked nickel metal-organic framework as highly efficient electrocatalysts for overall water splitting. Electrochim. Acta 297, 755-766 (2019). https://doi.org/10. 1016/j.electacta.2018.12.020

44. C. Huang, C. Pi, X. Zhang, K. Ding, P. Qin et al., In situ synthesis of MoP nanoflakes intercalated $\mathrm{N}$-doped graphene nanobelts from $\mathrm{MoO}_{3}$-amine hybrid for high-efficient hydrogen evolution reaction. Small 14(25), 1800667 (2018). https:// doi.org/10.1002/smll.201800667

45. X. Zhang, X. Yu, L. Zhang, F. Zhou, Y. Liang et al., Molybdenum phosphide/carbon nanotube hybrids as $\mathrm{pH}$-universal electrocatalysts for hydrogen evolution reaction. Adv. Funct. Mater. 28(16), 1706523 (2018). https://doi.org/10.1002/adfm. 201706523

46. Y. Huang, J. Ge, J. Hu, J. Zhang, J. Hao et al., Nitrogen-doped porous molybdenum carbide and phosphide hybrids on a carbon matrix as highly effective electrocatalysts for the hydrogen evolution reaction. Adv. Energy Mater. 8(6), 1701601 (2018). https://doi.org/10.1002/aenm.201701601

47. X. Yang, Y.C. Kimmel, J. Fu, B.E. Koel, J.G. Chen, Activation of tungsten carbide catalysts by use of an oxygen plasma pretreatment. ACS Catal. 2(5), 765-769 (2012). https://doi. org/10.1021/cs300081t

48. J. Xie, H. Zhang, S. Li, R. Wang, X. Sun et al., Defect-rich $\mathrm{MoS}_{2}$ ultrathin nanosheets with additional active edge sites for enhanced electrocatalytic hydrogen evolution. Adv. Mater. 25(40), 5807-5813 (2013). https://doi.org/10.1002/adma. 201302685

49. Z.Q. Liu, H. Cheng, N. Li, T.Y. Ma, Y.Z. Su, $\mathrm{ZnCo}_{2} \mathrm{O}_{4}$ quantum dots anchored on nitrogen-doped carbon nanotubes as reversible oxygen reduction/evolution electrocatalysts. Adv. Mater. 28(19), 3777-3784 (2016). https://doi.org/10.1002/ adma.201506197
50. C.C.L. McCrory, S. Jung, I.M. Ferrer, S.M. Chatman, J.C. Peters et al., Benchmarking hydrogen evolving reaction and oxygen evolving reaction electrocatalysts for solar water splitting devices. J. Am. Chem. Soc. 137(13), 4347-4357 (2015). https://doi.org/10.1021/ja510442p

51. A.T. Swesi, J. Masud, M. Nath, Nickel selenide as a high-efficiency catalyst for oxygen evolution reaction. Energy Environ. Sci. 9(5), 1771-1782 (2016). https://doi.org/10.1039/C5EE0 $2463 \mathrm{C}$

52. J. Zhong, J.J. Deng, B.H. Mao, T. Xie, X.H. Sun et al., Probing solid state $\mathrm{N}$-doping in graphene by X-ray absorption nearedge structure spectroscopy. Carbon 50, 335-338 (2012). https://doi.org/10.1016/j.carbon.2011.08.046

53. D. Pacile, M. Papagno, A.F. Rodriguez, M. Grioni, L. Papagno et al., Near-edge X-ray absorption fine-structure investigation of graphene. Phys. Rev. Lett. 101(6), 066806 (2008). https:// doi.org/10.1103/PhysRevLett.101.066806

54. C. Wang, D. Wang, S. Liu, P. Jiang, Z. Lin et al., Engineering the coordination environment enables molybdenum singleatom catalyst for efficient oxygen reduction reaction. J. Catal. 389, 150-156 (2020). https://doi.org/10.1016/j.jcat.2020.05. 034

55. Y. Lin, L. Yang, Y. Zhang, H. Jiang, Z. Xiao et al., Defective carbon-CoP nanoparticles hybrids with interfacial charges polarization for efficient bifunctional oxygen electrocatalysis. Adv. Energy Mater. 8(18), 1703623 (2018). https://doi.org/10. 1002/aenm.201703623

56. Y. Li, D. Liu, J. Gan, X. Duan, K. Zang et al., Sustainable and atomically dispersed iron electrocatalysts derived from nitrogen- and phosphorus-modified woody biomass for efficient oxygen reduction. Adv. Mater. Interfaces 6(4), 1801623 (2019). https://doi.org/10.1002/admi.201801623

57. D. Zhao, K. Sun, W.C. Cheong, L. Zheng, C. Zhang et al., Synergistically interactive pyridinic-N-MoP sites: identified active centers for enhanced hydrogen evolution in alkaline solution. Angew. Chem. Int. Ed. 59(23), 8982-8990 (2020). https://doi.org/10.1002/anie.201908760

58. X. Cui, P. Ren, D. Deng, J. Deng, X. Bao, Single layer graphene encapsulating non-precious metals as high-performance electrocatalysts for water oxidation. Energy Environ. Sci. 9(1), 123-129 (2016). https://doi.org/10.1039/c5ee03316k 